\title{
Milky Way and Magellanic Cloud Surveys for Planetary Nebulae
}

\author{
Q.A. Parker ${ }^{1,2}$, A. Acker ${ }^{3}$, D.J. Frew ${ }^{1}$ \\ and W.A. Reid ${ }^{1}$ \\ ${ }^{1}$ Department of Physics, Macquarie University \\ email: qap@ics.mq.edu.au \\ ${ }^{2}$ Anglo-Australian Observatory, NSW, 2102, Australia \\ ${ }^{3}$ Observatoire de Strasbourg, France
}

\begin{abstract}
The recent on-line availability of large-scale, wide-field surveys of the Galaxy and Magellanic Clouds in several optical and near/mid-infrared passbands has provided unprecedented opportunities to refine selection techniques and eliminate contaminants in PN surveys. This has been coupled with new surveys offering improved detection rates via higher sensitivity and resolution. This will permit more extreme ends of the PN luminosity function to be explored and enable studies of under represented PN evolutionary states. Known PNe in our Galaxy and LMC have thus been significantly increased over the last few years due primarily to the advent of narrow-band imaging in important nebula lines such as $\mathrm{H} \alpha$, [O III] and [S III]. These PNe are generally of lower surface brightness, larger angular extent, in more obscured regions and in later stages of evolution than those in most previous surveys. A more representative PN population for in-depth study is now available, particularly in the LMC where the known distance adds considerable utility for derived PN parameters. Future prospects for Galactic and LMC PNe research are briefly highlighted.
\end{abstract}

Keywords. surveys, catalogs, planetary nebulae: general.

\section{Introduction: Why survey for $\mathrm{PNe}$ ?}

Planetary Nebulae (PNe) are among the most complex, varied and fascinating of celestial phenomena. They hold the key to determining the physics and time-scales of mass loss and evolution for low mass stars (Iben 1995). They are vital probes of nucleosynthesis processes, abundance gradients and chemical enrichment of the interstellar medium. PNe act as powerful tracers of our Galaxy's star-forming history (e.g. Maciel \& Costa 2003) and are useful kinematical probes visible to large distances due to their rich emission-line spectrum. Timescales last for a mere 30,000-70,000 years (e.g. Zijlstra \& Pottasch 1991; Pierce et al. 2004; Frew 2006) so, though a common end point of stellar evolution, their short lifetimes limit numbers for study. This has in turn restricted scope for unraveling their full characteristics and evolutionary diversity. These scientific drivers and others motivate the search for and study of the PNe diaspora in our galaxy.

\section{Galactic PNe catalogues}

Galactic PNe catalogues represent an extremely heterogeneous collection from over 100 years of diverse searches undertaken with a host of different telescopes and detection techniques. They are primarily presented in the catalogue of galactic planetary nebulae by Perek \& Kohoutek 1967: PK67, which contains the distilled work of dozens of researchers over many decades. The Strasbourg/ESO Catalogue of Galactic PNe (SECGPN; Acker et al. 1992, Acker et al. 1996), is based on a spectrophotometric survey of all known PNe 
Table 1. History of Major Galactic PNe Compilations and Catalogues

\begin{tabular}{lcc}
\hline Investigators & Epoch & Number PNe \\
\hline Messier, Darquier, Mechain & $1764-1782$ & 4 \\
William Herschel & $\sim 1800$ & 20 \\
Curtis & 1918 & 78 \\
Vorontsov-Velyaminov, Parenago & 1931 & 121 \\
Vorontsov-Velyaminov & 1948 & 288 \\
Minkowski & 1950 & 371 \\
Perek, Kohoutek; CGPN & 1967 & 1036 \\
Kohoutek supplements & $1978-2000$ & 721 \\
Acker et al.; (SECGPN) & 1992 & $1143+347^{*}$ \\
Acker et al.; (SECGPN supp) & 1996 & $243+142^{*}$ \\
Kohoutek; CGPN updated & 2001 & 1510 \\
Parker et al.; MASH & 2003,2006 & $756+146^{*}$ \\
\hline
\end{tabular}

Note there is essentially no overlap in content between the new MASH PN and those in the Acker and Kohoutek compilations. The asterisked numbers refer to 'possible' PNe only.

found in PK67 but including all later discoveries. The SECGPN contains exhaustive data (mostly based on the authors' own observations) for 1385 true and probable PNe, 489 possible $\mathrm{PNe}$ and 330 misclassified objects. The updated compilation of Kohoutek (2001) listed a small number of additional new PNe. Additional Galactic PNe have been discovered in relatively small numbers since then until the advent of the MASH project (e.g. Parker et al. 2003, 2006).

The main Acker and Kohoutek references largely contain the same sources. However, special note should be made of the 'Innsbruck group' (e.g. Weinberger et al. 1983, Kerber et al. 1996) who discovered $\sim 120$ new PNe from painstaking visual examination of existing broad-band POSS and ESO/UK Schmidt photographic plate surveys over a twenty year period. These catalogues are often the starting point for large numbers of research programmes that seek to exploit individual PNe or small samples for more detailed study. Many PNe have several designations and form part of the samples of various researchers often discovered independently. There are now over 6000 papers with the phrase 'Planetary Nebulae' in the title from 1900-2005 (based on a search of the NASA/ADS abstract service) which attest to the activity in the field.

\section{Galactic and Magellanic Cloud PNe discovery techniques}

Historically, the mainstay of PNe discovery has been in the optical regime. A significant fraction of known Galactic and Magellanic Cloud PNe have come from dedicated searches using modest aperture but wide-field telescopes, required due to the very low number densities of PNe per square degree. These were often equipped with low dispersion objective-prisms (e.g. Henize 1967). Images were recorded on photographic exposures and visually examined. Later they were digitised for more convenient study. Narrow-band interference filters have also been used to good effect centred on strong PNe emission lines (e.g. [O III] and $\mathrm{H} \alpha$ ) often with contemporaneous on-band/off band exposures. Higher resolution slit spectroscopy is then used to confirm candidates found on both direct and objective-prism photographs. More recently such techniques have been extended to CCD mini-surveys and in other lines (e.g. [S III] in the NIR, Jacoby \& Van de Steene 2004).

Other programmes have made use of the specific IRAS colours of PNe candidates through the far-infrared excess associated with the warm dust in the nebulae and associated 6-cm radio continuum observations (e.g. Ratag \& Pottasch 1991, and Van de 


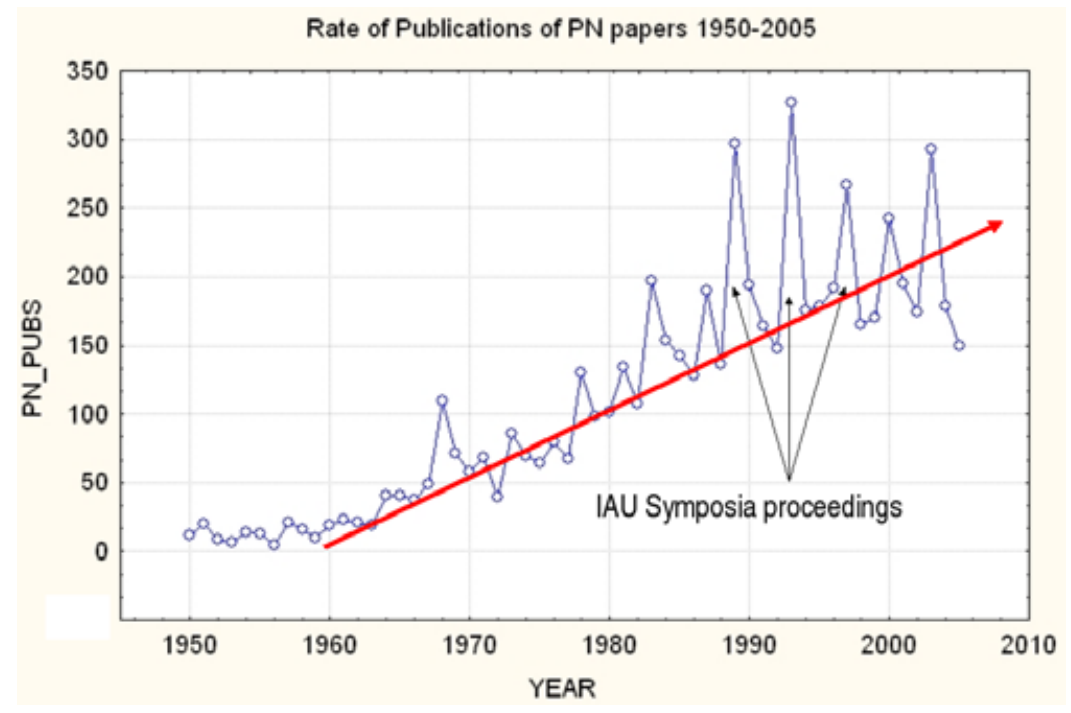

Figure 1. PNe paper publications since 1950

Steene \& Pottash, 1995 who identified 63 new and 67 possible bulge PNe). There is always a question mark over candidates selected purely by NIR colours until confirmatory spectroscopy and higher resolution optical/NIR or radio imaging are obtained. Use of mid-infrared wavelengths (e.g. MSX and Spitzer/GLIMPSE) can be used as corroborating data or as a way of eliminating candidates.

Nevertheless, until recently, progress in advancing Galactic and Magellanic Cloud PNe numbers has been slow and piecemeal, with papers dedicated to a few PNe discoveries still common. Several newer, targeted PN programmes are based on CCD narrowband imaging in regions like the Galactic bulge, where expected number densities are high. These have revealed significant new PNe over the relatively small areas that can be surveyed. However, the numbers remain modest, e.g. Beaulieu et al. (1999) with 56 new PNe from [O III] imaging and red follow-up spectroscopy and Jacoby \& Van de Steene 2004 with 94 bulge PNe from NIR imaging in the [S III] $9532 \AA$ emission line. In addition Boumis et al. (2003) found 25 bulge PNe from [O III] imaging and low resolution spectroscopy.

\section{Problems with generating representative $P N$ catalogues}

$\mathrm{PNe}$ are very heterogeneous in their intrinsic and observed properties, which causes problems in any effort to compile representative samples. They exhibit a wide variety of morphology, evolutionary state, angular/physical extent and expansion velocity with inherent variation in levels of excitation, central star progenitor mass, chemical composition, stratification and the presence (or more likely detectability) of AGB halos. The degree of interaction with and modification by the surrounding environment as a function both of environment and PN age is also a factor as is the level of intervening dust along the line of sight. Finally, there is the influence of possible companions (binary or sub-stellar components), magnetic fields and the interacting wind properties from the central star and resultant sculpting of shape. It is thus hardly a surprise that existing PNe catalogues are highly incomplete, undoubtedly biased and may not be representative of the true range of PNe types and fractions that exists. We need surveys which provide 

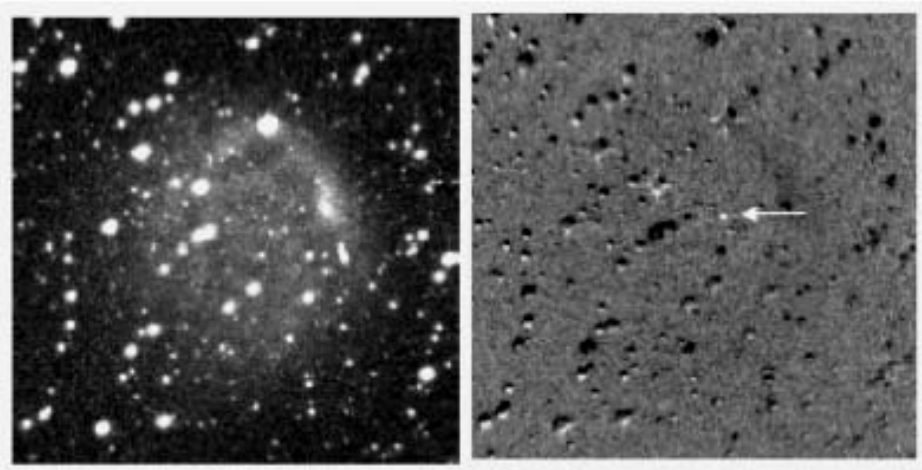

Figure 2. Identification of the central star candidate of PHR0942-5220 with $\mathrm{H} \alpha$ (left) and $\mathrm{B}_{j}$ - R difference image (right). Notice the off-center position of the central star $\left(\mathrm{B}_{j}=19.8\right)$ as indicated.

improved sky coverage, enhanced sensitivity and resolution, deep observations in suitable passband(s) and the acquisition of data relevant for combination with other imaging and spectroscopic data in other indicative regimes to validate $\mathrm{PNe}$ discoveries.

\subsection{Determining accurate $P N$ positions}

Determining accurate positions for known PNe has been problematic. Some PNe have been found and then lost (especially in the Magellanic Clouds) due to poor initial positions based on crude measures from old photographic plates. Published positions for PNe can vary markedly depending on: discovery epoch (and hence available technology at the time for measuring position), discovery medium/technique (e.g. passband), astrometric integrity/capability of telescope and detector combination. This is coupled with significant and inherent variety in PN size, shape and symmetry and as a function of waveband or emission line imaged. A subjective assessment of the nebula's apparent geometric centroid is often used, especially for large resolved and/or asymmetric nebulae and if no CSPN is identified. Improved imaging and sensitivity can reveal a fuller extent of many PNe and require an adjustment to published positions. If a CSPN has been identified, two positions may be required, one for the CSPN and one for the surrounding nebula, especially if the star has significant proper motion and is non-central, or if the visible nebula is asymmetric. Much work has lately gone in to trying to provide uniform co-ordinates for all known Galactic PNe (e.g. Kimeswenger 2001, Kerber et al., 2003).

\section{Non-PNe contaminants in catalogues}

A wide range of different astrophysical objects have turned up in previous catalogues of PNe and many contaminants undoubtedly remain. This is mainly due to morphological similarity and/or detection of PN-like spectral signatures. Such mistaken identity is often compounded by associated poor imaging, low spectral resolution, inadequate wavelength coverage and/or insufficient $\mathrm{S} / \mathrm{N}$ in the base discovery/confirmatory data. Kohoutek (1983) provides a good summary of PN mimics including: H II regions, supernova remnants (SNRs), reflection nebulosities, Wolf-Rayet shells, Herbig-Haro objects, symbiotic stars (and outflows) and low redshift emission-line galaxies. Acker \& Stenholm (1990) and Mikolajewska et al. (1997) identified 110 misclassified PNe and found 18 new symbiotic systems in previous compilations based on a careful analysis of new optical spectra they obtained. 


\subsection{Eliminating contaminants}

PNe exhibit characteristic features over a broad range of the electromagnetic spectrum. Ideally we want to combine multi-wavelength NIR, MIR, optical and radio imaging (including in narrow optical bands) with large spectral wavelength coverage in (UV), Optical/NIR to aid clean identification. Previously this was difficult to achieve without detailed individual follow-up. However, the advent of major wide-area surveys in the last few years has improved the situation considerably (e.g. SHS, SHASSA, IPHAS, MSX, MOST, NVSS). These surveys, accessible on-line, offer unprecedented opportunities not only for discovery and to permit investigation of relationships between measured and derived $\mathrm{PNe}$ parameters with unrivalled precision, but also as a mean of refining selection techniques and eliminating contaminants.

The use of diagnostic diagrams from measured optical line ratios using $\mathrm{H} \alpha$, [N II] and [S II] line strengths can be a useful discriminator (e.g. Sabbadin \& D'Odorico 1976, Sabbadin, Minello \& Bianchini 1977). The locations of known H II regions and SNRs generally occupy different regions of the line-ratio diagrams distinct from the PNe. However, the $[\mathrm{N}$ II $] / \mathrm{H} \alpha$ ratio alone must be used with caution as most higher-excitation $\mathrm{PNe}$ have relatively weak [N II] emission. Also, very high excitation PNe have no measurable [N II] or [S II] emission. If only red spectra are available for such an object, then other criteria (morphology, central star, environment) will be needed to classify it unambiguously.

A number of large, nearby (currently accepted) PNe may in fact be Strömgren spheres in the ISM ionised by a hot pre-white dwarf or subdwarf (the original PN having long since dissipated). These may have line ratios which mimic true PNe: e.g. Hewett 1 (Hewitt et al. 2003) has been shown by Chu et al. (2004) to probably not be a bona-fide PN. DeHt 5, Sh 2-174, and RE J1738+665 are also examples of such cases. Their true nature is currently being investigated by Madsen \& Frew (2006, in preparation) where high resolution WHAM velocity maps for large angular size PN can resolve ambiguity.

\subsection{The Galactic Bulge sample}

Many bulge emission sources are compact or even unresolved ( $\sim 8 \mathrm{kpc}$ away). Spectra alone can sometimes be insufficient if $\mathrm{S} / \mathrm{N}$ and wavelength coverage are inadequate. Ancillary information in terms of MSX imagery and 2MASS colours may be inconclusive or suffer from contamination due to crowding. Symbiotic stars (e.g. Belczyński et al. 2000) are one source of contaminants with many objects in both catalogue types! Symbiotics can be identified based on red DENIS IJKs and/or 2MASS JHKs colour indices (Schmeja \& Kimeswenger 2001; Larios \& Phillips 2005). Evidence for a red continuum in the optical spectrum, or strong [O III] $4363 \AA$ emission relative to $\mathrm{H} \alpha$ is also helpful (see Gutiérrez-Moreno, Moreno \& Cortés 1995). Nevertheless contamination in current catalogues remains a problem. There may well be a link between symbiotics and extreme bipolarity while true PNe may also have binary symbiotic companions.

\section{The MASH PNe survey of the Southern Galactic Plane}

Based on careful scrutiny of the high resolution, high sensitivity AAO/UKST Halpha Survey of the Southern Galactic plane, e.g. Parker et al. 2005 (and http://wwwwfau.roe.ac.uk/sss/halpha/), a significant new sample of Galactic PNe have been uncovered. The project is known as MASH (Macquarie/AAO/Strasbourg $\mathrm{H} \alpha \mathrm{PNe}$ ) and is the culmination of an extensive programme of visual identification and confirmatory spectroscopy between 1998 and 2005. Over 2500 individual spectra were obtained and 902 new PNe uncovered (Parker et al. 2003, Parker et al. 2006). The entire MASH sample has been derived from the same, uniform, narrow-band survey data yielding the largest, 


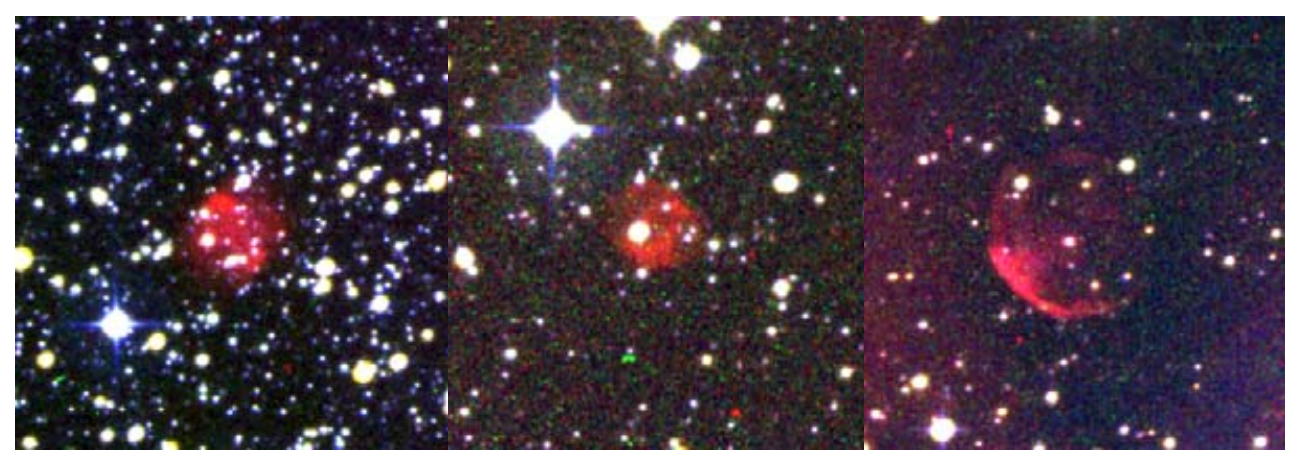

Figure 3. 3 MASH PNe consecutive in RA: PHR0924-5506, PHR0927-4347, PHR0928-4936; 3 arcmin regions, \& $\mathrm{H} \alpha$, SR, Bj RGB colour combination (courtesy B.Miszalski)

least biased and most homogeneous Galactic PNe sample over the widest evolutionary range. The sample is also less affected by dust and so many PNe are easier to detect than with [O III] imaging in more obscured regions of the Galactic Plane. MASH has increased the number of Galactic PNe by $\sim 60 \%$, but for a sample obtained only in the Southern Galactic plane. MASH should have a significant impact on $\mathrm{PNe}$ research, especially at the faint end of the PNe luminosity function. Improvements of the kinematics and the dynamics of the galactic bulge are promised thanks to $\sim 470$ new MASH bulge PNe (e.g. Peyaud 2005, and Peyaud et al. this volume).

\section{The Future of Galactic PNe surveys}

In the northern hemisphere the IPHAS $\mathrm{H} \alpha$ survey project on the INT (Drew et al. 2005), will be complete by Dec 2007. This promises to do for Northern PNe what MASH has accomplished in the South (e.g. Corradi et al. 2005). VPHAS is another H $\alpha$ survey that will start on the VST in Chile in late 2006 and should be complete by 2010 (J.Drew PI). All these surveys should considerably increase Galactic PNe, coupled with better exploitation of all existing multi-wavelength resources to refine selection.

\subsection{The distance problem for Galactic PNe}

A key problem with Galactic PNe and their study has been in measuring an accurate distance (see Pottasch 1996 for a review). Almost every quantity of interest for PNe, such as physical extent of inner shell and outer halo, total nebular mass, luminosity and evolutionary states of central stars, depends on knowing their distances (e.g. Ciardullo et al. 1999). The problem is exacerbated by the intrinsic faintness of $\mathrm{PN}$ outer halos and biases introduced by variable Galactic extinction. Progress requires refinement of key measured PN parameters (e.g. Frew \& Parker, this volume) or a homogeneous sample of PNe and their halos in an entire galactic system at known distance, and sufficiently close that the PNe can be resolved and studied with currently available technology.

\section{PNe studies in the Magellanic Clouds}

Since the distances to the Magellanic Clouds have been well determined (e.g. Nelson et al. 2000) all MC PNe can be effectively considered as co-located ( $50 \mathrm{kpc}$ for the LMC). This permits direct estimation of Magellanic Cloud PNe physical parameters. With the HST and a new generation of large aperture telescopes this can also now be done for their central stars. LMC/SMC PNe are thus absolute probes of stellar and 
Table 2. LMC PNe Surveys

\begin{tabular}{lcl}
\hline Investigators & Epoch & Number PNe discovered \\
\hline Henize & 1956 & 97 \\
Lindsay & 1963 & 65 \\
Westerlund \& Smith & 1964 & 42 (100 sq.deg) \\
Sanduleak, MacConnell \& Phillip & 1978 & 102 (most of LMC) \\
Jacoby & 1980 & 41 (faint central region) \\
Sanduleak & 1984 & 25 \\
Morgan \& Good & 1992 & 86 (UKST obj.prism) \\
Morgan & 1994 & 54 (UKST obj.prism) \\
Reid \& Parker & 2006 & 471 (H $\alpha$ stack) \\
\hline
\end{tabular}

nebular brightness and nebular size. Also dimming of their light by intervening gas and dust is low and uniform (Kaler \& Jacoby 1990). The LMC/SMC each provide a single system in which the PN mass loss history, and hence that of intermediate to low mass stars, can be studied in detail. The current SMC census and a brief review of previous SMC PN surveys is described by Jacoby \& de Marco (2002). Here we concentrate on the LMC.

\subsection{Problems with previous LMC catalogues}

LMC/SMC PNe searches have historically been biased via strong selection effects as well as identification and astrometric problems. After Henize (1956), subsequent H $\alpha$ surveys were progressively deeper through better instrumental resolutions and observational configurations. This improved sensitivity resulted in an increased number of emissionline detections. However, surveys continued to use objective-prism plates and separated emission-line stars from nebulae according to whether a continuum could be seen adjacent to the $\mathrm{H} \alpha$ line. Also, the deeper surveys do not cover the full 25 square degree region occupied by the main bar of the LMC. Samples thus comprise a heterogeneous compilation from surveys with varying depth, selection technique, detection efficiency and spatial coverage. This makes them of limited use for the estimation of key PN parameters necessary to study their evolution, that of their host galaxy and the associated inter-stellar medium enrichment and mass loss history. This demonstrates the need for a homogeneous, deep, well constructed LMC PN census with high astrometric integrity.

\subsection{A new LMC PN population uncovered}

The previous census of LMC PNe comprised $~ 300$ objects compared to the few thousand that might be expected (e.g. $\sim 1000$ Jacoby 1980). However, via a deep, $\sim 1$ arcsec resolution $\mathrm{H} \alpha$ LMC survey, Reid \& Parker (2006a,b) have uncovered 471 new PNe over the central 25 square degrees, where only 171 were previously known. This more than triples the number in the region accrued from all sources during the previous 80 years. As for MASH all new PNe originate from the same uniform observational data and have been followed-up spectroscopically using $2 \mathrm{dF}$ on the AAT at both low and medium resolution. This sample represents the largest and most homogeneous sample of spectroscopically confirmed PN in any galactic system.

\section{Conclusions}

Over the last few years there has been a new golden age of PNe discovery in our Galaxy and the Magellanic clouds. This has been largely due to the advent of powerful new, wide-field, narrow-band optical surveys in the southern and northern hemispheres, complemented by the online availability of multi-wavelength imaging surveys in the radio, 
NIR and MIR. Together, these surveys, and their accessibility, offer a powerful combination for discovery and comparison. New insights into the evolution, environmental influences and fundamental characteristics of this fascinating phenomenon are promised.

\section{References}

Acker, A. \& Stenholm, B. 1990, A\&SAS, 86, 219

Acker, A. et al. 1992, Strasbourg-ESO Catalogue of Galactic PNe

Acker, A. et al. 1996, First Supplement to the SECPGN. Observatoire de Strasbourg

Beaulieu, S.F., Dopita, M.A. \& Freeman K.C. 1999, ApJ, 515, 610

Belczyński, K. et al. 2000, A\&SAS, 146, 407

Boumis, P., Paleologou, E.V., Mavromatakis, F. \& Papamastorakis, J. 2003, MNRAS, 339, 735

Ciardullo, R. et al. 1999, AJ, 118, 488

Chu, Y.-H., Gruendl, R.A., Williams, R.M., Gull, T.R. \& Werner, K. 2004, AJ, 128, 2357

Corradi, R. et al. 2005, AIP Conf.Ser. 804, eds R.Szczerba, G.Stasinska \& S.K.Gorny, p.7

Drew, J., et al. 2005, MNRAS, 362, 753

Frew, D.J. 2006, PhD thesis, Macquarie University, Sydney

Gutiérrez-Moreno, A., Moreno, H. \& Cortés, G. 1995, PASP, 107, 462

Henize, K.G. 1956, ApJS, 2, 315

Henize, K.G. 1967, ApJS, 14, 125

Hewitt, P.C. et al. 2003, ApJ, 599, 37

Iben., I.J. 1995, Phys. Reports, 250, 2

Jacoby, G. 1980, AJS, 42, 1

Jacoby, G.H. \& de Marco, O. 2002, AJ, 123, 269

Jacoby, G.H. \& van de Steene G. 2004, A\& A, 419, 563

Kaler, J. \& Jacoby, G. 1990, ApJ,362,491

Kerber, F., Lercher, F. \& Weinberger, R. 1996, A\&AS, 119, 423

Kerber, F., Mignani, R., Guglielmetti, F. \& Wicenec, A. 2003, A\&A, 408, 1029

Kimeswenger, S., 2001, RMxAA, 37, 115

Kohoutek, L. 1983, Proc. IAU Symp.103, 'Planetary Nebulae', ed. D. R. Flower, p. 17

Kohoutek, L. 2001, Catalogue of Galactic Planetary Nebulae (Updated Version 2000). Abhandlungen Hamburger Sternwarte, XII

Larios, G.R. \& Phillips J.P. 2005, MNRAS, 357, 732

Nelson, C. et al. 2000, AJ,119,1205

Maciel, W.J. \& Costa, R.D.D. 2003, IAU Symp. 209, ASP Conf. Ser, eds M.Dopita et al., p.551

Mikolajewska, J., Acker, A. \& Stenholm, B. 1997, A\&̈A, 327, 191

Parker, Q.A. et al. 2003, IAU Symp.209, ASP.Conf.Ser, eds M.Dopita et al., p. 41

Parker, Q.A.et al. 2005, MNRAS, 362, 689

Parker, Q.A. et al. 2006, MNRAS, in press

Perek, L. \& Kohoutek, L. 1967, Catalogue of Galactic Planetary Nebulae, Publ. House Czech. Acad. Sci., no 3, 1-276

Peyaud, A.E.J. 2005, PhD thesis, Macquarie University, Sydney and ULP, Strasbourg.

Pierce, M.J., Frew D.J., Parker Q.A., \& Köppen J. 2004, PASA, 21, 334

Pottasch, S.R. 1996, A\&\&A, 307, 561

Ratag, M.A. \& Pottasch S.R. 1991, A\&SAS, 91, 481

Reid, W.A \& Parker, Q.A. 2006, MNRAS, 365, 401

Reid, W.A \& Parker, Q.A. 2006, MNRAS, in press (astro-ph/0610152)

Riesgo-Tirado, H. \& López J.A. 2002, Rev. Mexicana AyA, 12, 174

Sabbadin, F. \& D'Odorico, S. 1976, A\& A, 49, 119

Sabbadin, F., Minello, S. \& Bianchini, A. 1977, A\& $A, 60,147$

Schmeja, S. \& Kimeswenger, S. 2001, A\&A, 377, L18

Van de Steene, G. C., \& Pottash, S. R., 1995, A\& $A$, 299, 238

Weinberger, R., Dengel, J., Hartl, H \& Sabbadin, F. 1983 ApJ, 265, 249

Zijlstra, A.A. \& Pottasch, S.R. 1991, A\&A, 243, 478 